\title{
Assessment of Clinical Impact in the Application of Chang Attenuation Correction to Lung Ventilation/Perfusion SPECT
}

\author{
Sina Izadyar ${ }^{1}$, Sassan Saber $^{2}$, and Ali Gholamrezanezhad ${ }^{3}$ \\ ${ }^{I}$ Department of Nuclear Medicine, Imam Khomeini Complex Hospital, Tehran University of Medical Sciences, Tehran, Iran; \\ ${ }^{2}$ Department of Pulmonary Disorders, Shariati Hospital, Tehran University of Medical Sciences, Tehran, Iran; and ${ }^{3}$ Research Institute \\ for Nuclear Medicine, Tehran University of Medical Sciences, Shariati Hospital, Tehran, Iran
}

The presence of a highly nonuniform distribution of attenuating tissues in the thorax may lead to attenuation artifacts and inaccuracies in reconstructed lung ventilation/perfusion SPECT images. The Chang algorithm is an easily applicable and accessible method of attenuation correction. The advantages inherent in the technique prompted us to evaluate the effect of using Chang attenuation correction on the quality of lung SPECT images and the clinical impact on interpretation. Methods: Lung scintigraphy started with planar and SPECT ventilation scans using ${ }^{81 \mathrm{~m} K r}$. A few minutes after the ventilation scintigraphy, planar and SPECT perfusion studies using 99mTc-macroaggregated albumin were performed. The Chang algorithm was applied for attenuation correction. Afterward, standard SPECT processing was performed on the images, leading to images in the horizontal, coronal, and sagittal planes. The same studies were also processed to the same planes without application of attenuation correction. Finally, all scintigraphic images were reviewed by 2 nuclear medicine academic clinicians, and a final diagnosis was reached by consensus, categorizing the studies into 3 groups—normal, low/intermediate, and high probability - for pulmonary embolism. Results: The study included 45 patients (16 men and 29 women) with a mean age of $50.0 \mathrm{y}$. Between the 2 noncorrected and attenuation-corrected readings, 16 diagnoses remained the same. However, after attenuation correction, 13 patients were moved to lower categories (i.e., from high probability to normal or low/ intermediate probability or from low/intermediate probability to normal) and 16 patients were moved to higher categories (i.e., from low/intermediate probability to high probability or from normal to low/intermediate or high probability). The difference between noncorrected and attenuation-corrected readings was statistically significant $(P<0.01)$. Conclusion: After attenuation correction, subjective image quality and sharpness improved, leading in some cases to increased confidence on the part of the readers. The Chang attenuation correction method may have the potential to be considered as an alternative approach toward attenuation correction in those situations in which the additional radiation burden of CT is not acceptable.

Received Dec. 8, 2010; revision accepted May. 24, 2011.

For correspondence or reprints contact: Ali Gholamrezanezhad, Research Institute for Nuclear Medicine, Shariati Hospital, Tehran University of Medical Sciences, North Karegar Ave., Tehran, 14114, Islamic Republic of Iran. Published online Sep. 19, 2011.

COPYRIGHT @ 2011 by the Society of Nuclear Medicine, Inc.
Key Words: lung perfusion; lung ventilation; single photon emission computed tomography; Chang's attenuation correction J Nucl Med Technol 2011; 39:290-294 DOI: 10.2967/jnmt.110.086470

D espite the rapidly growing application of competing diagnostic imaging modalities (most importantly spiral CT pulmonary angiography [CTPA]), lung perfusion and ventilation scintigraphy is yet frequently used for noninvasive diagnosis of pulmonary embolism (PE) (1). Remarkable advantages inherent in the technique, including low radiation dose to the patient, many years of experience with its use, widespread availability, and relatively low cost, have overcome the obstacles and challenges that perfusion and ventilation scintigraphy face, such as slightly lower diagnostic accuracy compared with CTPA. However, CTPA is not free of drawbacks and shortcomings: Radiation- and contrast-related complications during CTPA are important problems, particularly to women, younger patients, those with underlying renal disorders, and all who undergo repeated examinations. More important, contrary to the prevailing belief, the diagnostic accuracy of CTPA in PE is not absolute. In smaller, more distal PEs, the positive predictive value of CTPA drops from $97 \%$ to less than $70 \%$ for a segmental vessel and almost $25 \%$ for a subsegmental branch, illustrating its poor accuracy in these common settings (2).

Interest in applying SPECT for perfusion and ventilation scanning is increasing. Previous studies have shown that fast perfusion and ventilation SPECT imaging with standard doses of radiotracers is feasible and provides good-quality images $(1,2)$. As the main advantage, the application of SPECT allows for the detection of subsegmental defects more accurately than do conventional planar views, and higher sensitivity, specificity, and interobserver agreement for lung studies have been proposed as the remarkable benefits of SPECT over conventional planar imaging $(1,3)$. However, the presence of the highly nonuniform distribution 
of attenuating tissues in the thorax may lead to attenuation artifacts (due to scattering and absorption of $\gamma$-photons) and inaccuracies in reconstructed images (1). Also, attenuation can lead to hot rim artifacts in reconstructed views if not corrected for. In fact, photon attenuation is one of the primary causes of artifacts in all SPECT procedures (e.g., cardiac, brain, and lung studies) $(4,5)$. The unpleasant end result of attenuation is that fewer photons are detected from structures deeply located in the body than from superficial tissues, potentially creating artifacts mimicking perfusion or ventilation defects.

To overcome this problem, several attenuation correction algorithms have been proposed. The method of Chang (6) is one of the most easily and widely used for attenuation correction and is supplied with most commercial $\gamma$-cameras. The Chang method simply calculates the regular attenuation for photons traveling from each point in the body at different angles and hence requires some assumptions to be made about the degree of attenuation and the distribution of activity. Although the method is only an approximate correction, it is confirmed to be quantitatively equivalent, with comparable fast calculation times, to other iterative direct approaches (4). Its advantages, including accessibility and fast processing (1), along with reports confirming the clinical impact of attenuation correction in other thoracic SPECT imaging (7), prompted us to evaluate the effect of using the Chang attenuation correction method on the quality of lung SPECT images and its impact on the interpretation and final diagnosis.

\section{MATERIALS AND METHODS}

Recruitment of patients began in October 2008 and ended in May 2009. All consecutive patients who were referred for the assessment of possible PE were investigated through ${ }^{99 \mathrm{~m} T c-m a c r o a g g r e g a t e d ~ a l b u m i n ~\left({ }^{9} \mathrm{~m} T c-M A A\right)}$ lung perfusion scintigraphy. The scintigraphic studies were done at the request of the referring physician, with no additional intervention on the subjects. After the patients had been interviewed and had given verbal consent, their medical records and clinical, laboratory, and imaging investigations, including electrocardiography, transthoracic echocardiography, Doppler sonography of the lower-extremity veins, and D-dimer levels, were evaluated and recorded. The study was approved by the Committee on Ethics from the Research Institute for Nuclear Medicine, Tehran University of Medical Sciences.

\section{Imaging Protocol}

Lung scintigraphy started with planar and SPECT ventilation scans conducted during quiet tidal inhalation of ${ }^{81 \mathrm{~m}} \mathrm{Kr}, 600 \mathrm{MBq}(16.22 \mathrm{mCi})$, by an $\mathrm{O}_{2}$ flow of $1 \mathrm{~L} / \mathrm{min}$. A few minutes after ventilation scintigraphy, with the patients remaining in the same supine position, planar and SPECT perfusion studies using ${ }^{99 \mathrm{~m}}$ Tc-MAA were performed. A commercial ${ }^{99 \mathrm{~m}} \mathrm{Tc}-\mathrm{MAA}$ preparation (Atomic Energy Organization of Iran) was used. The labeling and quality control procedures were performed according to the manufacturer's instructions. In accordance with our institutional protocol, a dose of $185 \mathrm{MBq}(5.0 \mathrm{mCi})$ of ${ }^{99 \mathrm{~m}} \mathrm{Tc}-$ MAA was injected intravenously with the patients supine and spontaneously breathing as regularly as possible. The patients carefully maintained their supine position during image acquisition. Immobilization lasting $20 \mathrm{~min}$ was well tolerated by all patients. None needed to be in a sitting position for image acquisition.

All patients underwent $360^{\circ}$ SPECT of the thorax using a dual-head $\gamma$-camera (Genesis; ADAC) equipped with lowenergy high-resolution collimators and a $20 \%$ energy window centered at $140 \mathrm{keV}$ for the ${ }^{99 \mathrm{~m}} \mathrm{Tc}-\mathrm{MAA}$ perfusion studies and $190 \mathrm{keV}$ for the ${ }^{81 \mathrm{~m}} \mathrm{Kr}$ ventilation studies. Both the ventilation and the perfusion scans were acquired using a $128 \times 128$ matrix. A $180^{\circ}$ rotation per head was done in $20 \mathrm{~s}$ per step (36 steps). Images were reconstructed using a standard filtered backprojection algorithm with a Butterworth filter (cutoff frequency, 0.2; order, 8).

As the next step, attenuation correction was performed by applying the iterative reconstruction algorithm provided by the system. In previous experiments on the chest for technetium-energy studies, the broad-beam attenuation coefficient for water $\left(0.13 \mathrm{~cm}^{-1}\right)$ had been applied to regions designated to be soft tissue-that is, the entire volume within the outer body contour, excepting the lungs. A value of $0.047 \mathrm{~cm}^{-1}$ had been applied to the lungs (7). To simplify and make the method more easily applicable, however, we applied an attenuation coefficient of $0.09 \mathrm{~cm}^{-1}$ as the mean value for the thorax. This assumption is not supported by the literature and was considered as a way to make the attenuation correction method more applicable in routine clinical practice by decreasing its computational complexity. After attenuation correction, standard SPECT processing was performed on the images, producing images in the horizontal, coronal, and sagittal planes. Subsequently, the same studies were processed to the same planes without application of attenuation correction.

Finally, 2 nuclear physicians (both with more than $15 \mathrm{y}$ of academic clinical experience), unaware of which images had undergone attenuation correction, interpreted the SPECT data independently. In cases of disagreement, a final diagnosis was reached by consensus after discussion. Two sets of images were read in 2 different sessions 1 mo apart. The order of readings (regarding attenuation-corrected and non-attenuation-corrected images) was randomized. Because the diagnostic criteria for interpretation of SPECT for PE are not properly validated, the criteria proposed by Touya et al. (8) and applied clinically by Hata (9) were followed in our study (i.e., segmental defect visualization in at least 2 of 3 planes on the SPECT study was considered as evidence of PE), and the studies were categorized into 3 groups: normal, low/intermediate, and high probability of PE.

\section{Statistical Analysis}

Statistical analysis was done using the SPSS software package, version 17.0 (SPSS Inc.). The results were 
compared using the $\chi^{2}$ test. A $P$ value of less than 0.05 was considered statistically significant.

\section{RESULTS}

The study included 45 patients ( 16 men and 29 women), aged $23-85$ y with a mean $( \pm S D)$ of $50.0 \pm 5.9$ y. The consensus interpretation for noncorrected and attenuationcorrected SPECT images is presented in Table 1. Between the 2 noncorrected and attenuation-corrected readings, 16 diagnoses remained the same. However, after attenuation correction, 13 patients were moved to lower categories (i.e., from high probability to normal or low/intermediate probability or from low/intermediate probability to normal) and 16 patients were moved to higher categories (i.e., from low/intermediate probability to high probability or from normal to low/intermediate or high probability). The difference between noncorrected and attenuation-corrected readings was statistically significant $(P<0.01)$.

For example, in one of the patients attenuation correction eliminated an artifactual defect in the upper and middle lobes of the right lung, and therefore the high-probability interpretation was changed to an intermediate-probability interpretation (Fig. 1).

\section{DISCUSSION}

Previous studies have reported that attenuation correction improves defect-to-normal ratios in lung SPECT studies, potentially enhancing the diagnostic capability of the scintigraphy (1). Our study observations support such a conclusion: after attenuation correction, image quality and sharpness of the images improved in some cases (Fig. 1) but not in others (Fig. 2 ). When the quality and sharpness of the images were improved, the readers had increased confidence in their interpretation. Also, the number of studies with normal findings decreased and the number with high-probability findings increased. It seems that, because of attenuation artifact effects on noncorrected images, some defects are either missed or underestimated by the readers. Essential to avoid or minimize the number of misdiagnoses is that the activity distribution be accurately imaged without interfering artifacts (10-15).

TABLE 1

Final Interpretation of Ventilation/Perfusion Lung SPECT Studies Before and After Application of Chang Attenuation Correction

\begin{tabular}{lcc}
\hline Interpretation & $\begin{array}{c}\text { Noncorrected } \\
\text { readings }\end{array}$ & $\begin{array}{c}\text { Attenuation-corrected } \\
\text { readings }\end{array}$ \\
\hline Normal & 16 & 12 \\
Low or & 22 & 24 \\
intermediate & & \\
probability of & & \\
PE & 7 & 9 \\
High & \\
probability & & \\
of PE & & \\
\hline
\end{tabular}

As clearly stated by Núñez et al., it is not common in daily practice to perform attenuation correction on lung SPECT studies. This is due partly to the complexity of the available attenuation correction techniques, which are laborious, and probably also to the belief that any attenuation effects would equally affect both ventilation and perfusion images and prevent an observable mismatch mimicking PE (1). However, others have emphasized that SPECT requires correction for the image-degrading effects of photon attenuation and scatter, particularly when quantitative measures are required (16).

Based on our study findings, the overall benefit of attenuation correction in a clinical setting can be greater than anticipated. As expected by Núñez et al., limited benefit was achieved in cases with large or multiple ventilation/ perfusion mismatch defects interpreted as a high probability of PE. On the other hand, the ability to identify small perfusion defects is positively affected by attenuation correction, improving the sensitivity of SPECT studies of PE and the homogeneity of images $(1,14,15)$, as supported by our study, too. In our opinion, the advantages of attenuation correction are not limited to quantitative studies; qualitative interpretation of lung scintigraphy can also be affected. Previous studies have confirmed that attenuation correction significantly improves diagnostic accuracy in cardiac perfusion studies (17). However, before any conclusion can be made in lung SPECT studies, the findings should be confirmed in larger samples of patients, considering CTPA, angiography, or long-term follow-up as the gold standard. These were the main limitations of our study, in fact.

Regarding the general consensus on the adverse effects of attenuation on the diagnostic accuracy and image quality of scintigraphic studies, frequent efforts have been made to overcome the problem. Although different approaches have been examined, none is free of drawbacks: Attenuation correction using CT, although having the highest success in correcting artifacts and in increasing the specificity and accurate depiction and localization of disease, suffers from the disadvantage of added radiation burden to patients (18). In some situations, such an additional radiation dose is not acceptable (e.g., pregnant women, younger patients, patients undergoing repeated diagnostic studies for followup, and even premenopausal women regarding the risk of breast cancer (1)). Hence, several approximate attenuation correction techniques have been examined that lack the potential hazard of added radiation exposure. Generally, these approaches can be classified into 3 categories-preprocessing, iterative techniques, and postprocessingwhich are well reviewed by Sayed et al. (10). Again, none of these is perfect, and the advantages of any technique need to be weighed against its disadvantages and limitations. For example, the Sorenson method of attenuation correction has the advantages of being less sensitive with linear attenuation coefficient values and of giving lower SDs than the Chang method (10). However, in comparison, the Chang attenuation correction method works better than 


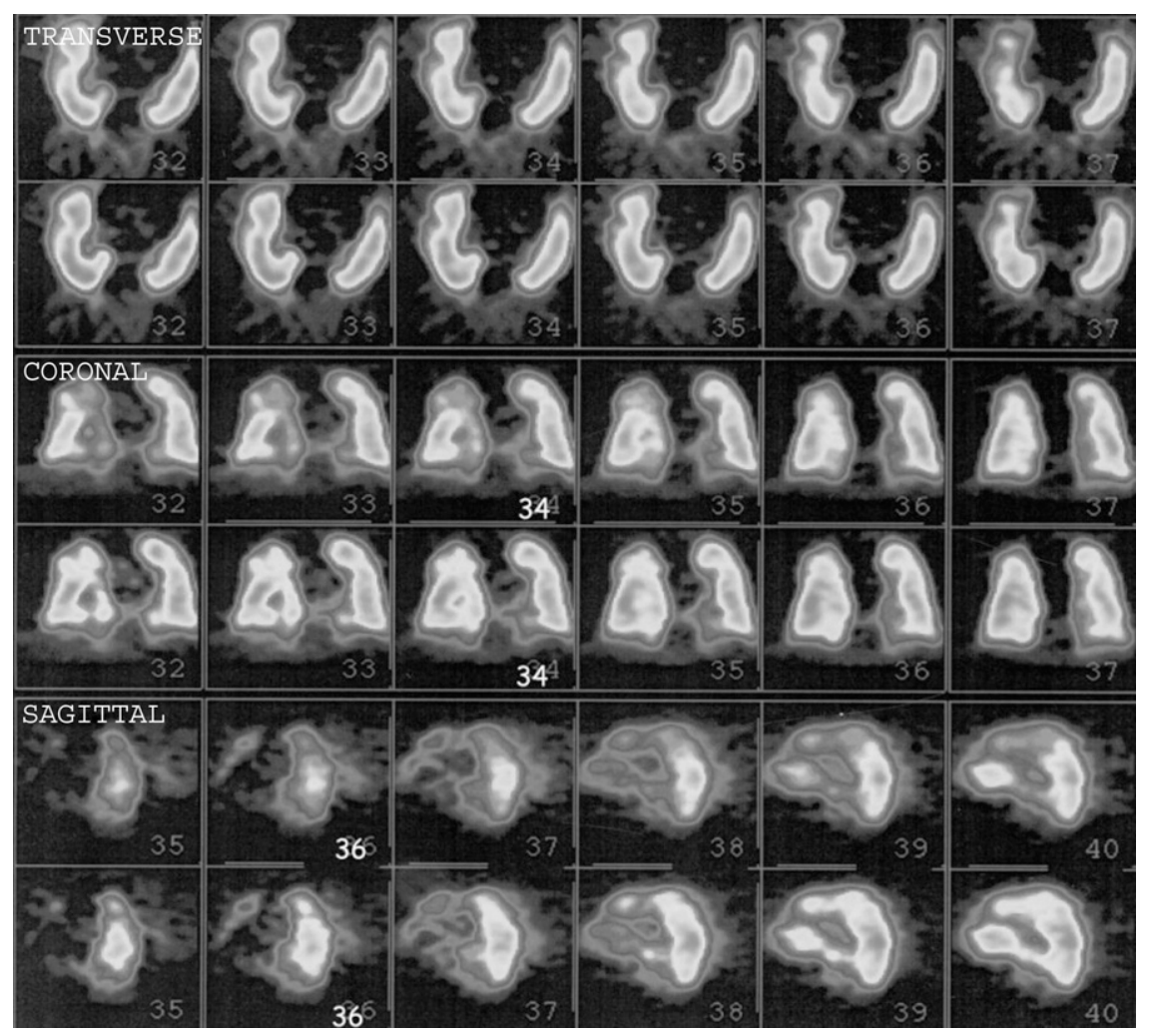

FIGURE 1. Lung perfusion SPECT planes before (top rows) and after (bottom rows) attenuation correction using Chang method. In this patient, quality and sharpness of images improved significantly. High-probability interpretation changed to intermediate probability. the Sorenson method in terms of the linearity in measured counts in hot regions. Generally, the Chang algorithm leads to relatively more quantitatively accurate outputs than the Sorenson technique (10).
This study had some limitations. We did not correlate SPECT-derived diagnoses with any other imaging modality, such as CTPA or pulmonary catheterization, as it was considered unethical to pose patients' with the risks and

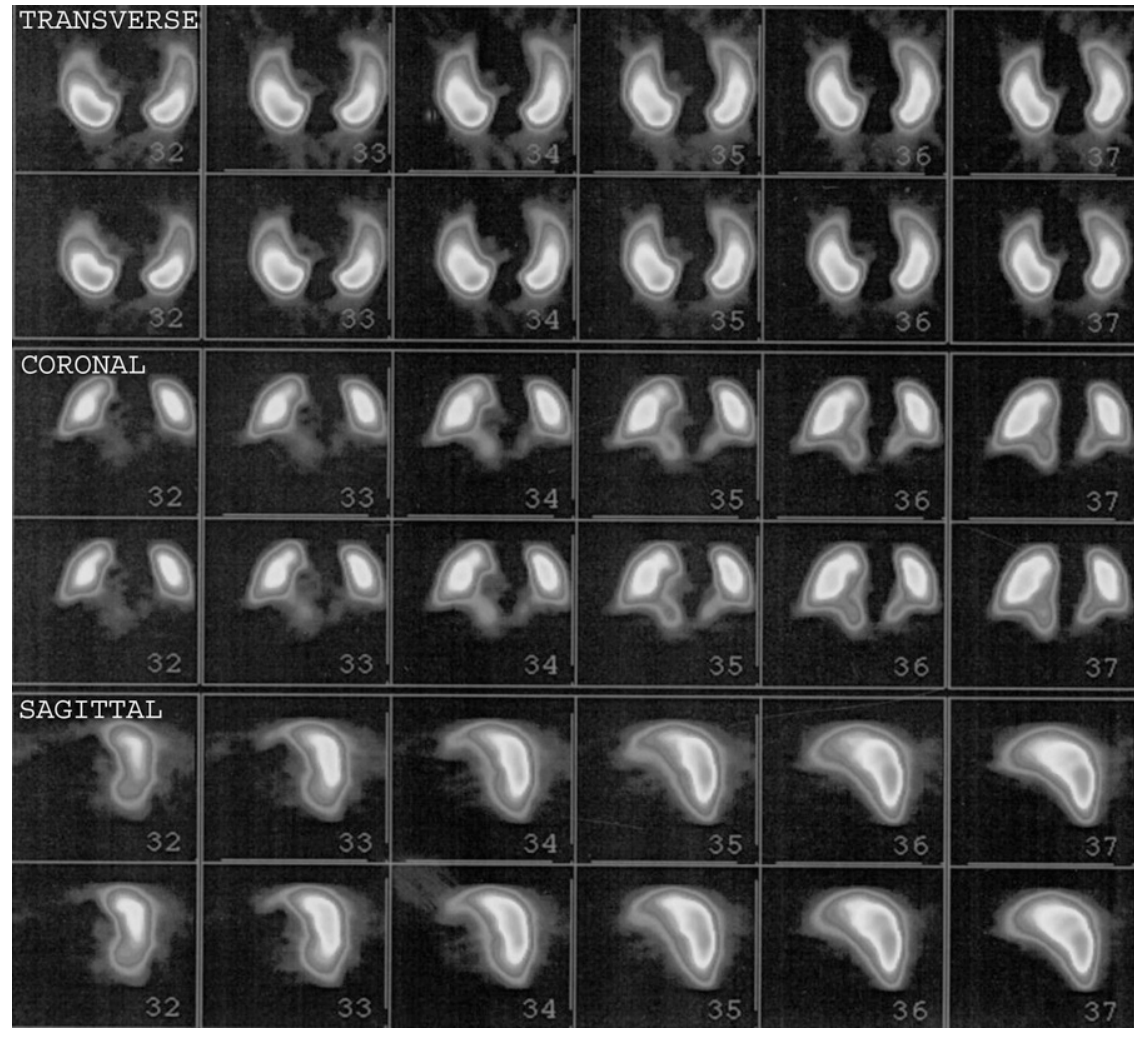

FIGURE 2. Lung perfusion SPECT planes before (top rows) and after (bottom rows) attenuation correction using Chang method. In this patient, quality and sharpness of images did not change appreciably. 
radiation inherent in these techniques just for a technical attenuation correction study. However, comparison with a gold standard is required in future studies, as it is important to evaluate whether attenuation correction creates artifactual defects. In our patients, we did not observe any new perfusion defects after application of a uniform attenuation coefficient of $0.09 \mathrm{~cm}^{-1}$; however, future studies should assess the accuracy of such a simple attenuation correction method, compared with the routine technique of differential attenuation correction using coefficients of $0.13 \mathrm{~cm}^{-1}$ for soft tissue and $0.047 \mathrm{~cm}^{-1}$ for lungs.

Lack of prospective follow-up of patients to evaluate the significance of altered interpretation on their outcome and clinical management was another major study limitation. Such follow-up needs to be included in future studies. Future studies should also consider quantitative analysis for more accurate assessment of the effects of Chang attenuation correction.

\section{CONCLUSION}

Attenuation-corrected lung ventilation/perfusion SPECT images can be obtained using the Chang method without the need for transmission scanning. Attenuation correction seems to change the final interpretation of lung SPECT studies significantly, decreasing the number of studies with normal findings and increasing the confidence of the interpreters in some cases. This method can be considered an easily applicable technique of attenuation correction when additional radiation burden from $\mathrm{CT}$ is not acceptable. However, the value of the altered interpretation should be evaluated in future studies.

\section{ACKNOWLEDGMENT}

No potential conflict of interest relevant to this article was reported.

\section{REFERENCES}

1. Núñez M, Prakash V, Vila R, Mut F, Alonso O, Hutton BF. Attenuation correction for lung SPECT: evidence of need and validation of an attenuation map derived from the emission data. Eur J Nucl Med Mol Imaging. 2009;36:1076-1089.

2. Schattner A. Computed tomographic pulmonary angiography to diagnose acute pulmonary embolism: the good, the bad, and the ugly. Arch Intern Med. 2009;169:1966-1968.

3. Gutte H, Mortensen J, Jensen CV, et al. Comparison of V/Q SPECT and planar V/Q lung scintigraphy in diagnosing acute pulmonary embolism. Nucl Med Commun. 2010;31:82-86.

4. Dey D, Slomka PJ, Hahn LJ, Kloiber R. Comparison of ordered subsets expectation maximization and Chang's attenuation correction method in quantitative cardiac SPET: a phantom study. Nucl Med Commun. 1998;19:1149-1157.

5. Mizuno T, Takahashi M, Kinda A. Evaluation of an attenuation correction method in brain perfusion single photon emission computed tomography using magnetic resonance imaging. Nippon Hoshasen Gijutsu Gakkai Zasshi. 2010;66:201-210.

6. Chang LT. A method for attenuation correction in radionuclide computed tomography. IEEE Trans Nucl Sci. 1978;25:638-643.

7. Wallis JW, Miller TR, Koppel P. Attenuation correction in cardiac SPECT without a transmission measurement. J Nucl Med. 1995;36:506-512.

8. Touya JJ, Corbus HF, Savala KM, Habibe MN. Single photon emission computed tomography in the diagnosis of pulmonary thromboembolism. Semin Nucl Med. 1986;16:306-336.

9. Hata T, Ikeda M, Nakamori S, et al. Single-photon emission computed tomography in the screening for postoperative pulmonary embolism. Dig Dis Sci. 2006;51:2073-2080.

10. Sayed IS, Zakaria A, Nik N. Comparison of Chang's with Sorenson's attenuation correction method by varying linear attenuation coefficient values in Tc-99m SPECT imaging. In: Gao X, Müller H, Loomes MJ, Comley R, Luo S, eds. Medical Imaging and Informatics. Berlin, Germany: Springer-Verlag; 2008:216-222.

11. Hayashi M, Deguchi J, Utsunomiya K, et al. Comparison of methods of attenuation and scatter correction in brain perfusion SPECT. J Nucl Med Technol. 2005;33:224-229.

12. Kim KM, Varrone H, Watabe H, et al. Contribution of scatter and attenuation compensation to SPECT images of nonuniformly distributed brain activities. J Nucl Med. 2003;44:512-519.

13. Zaidi H, Hasegawa B. Determination of the attenuation map in emission tomography. J Nucl Med. 2003;44:291-315.

14. Gustafsson A, Bake B, Jacobsson L, Johansson A, Ljungberg M, Moonen M. Evaluation of attenuation corrections using Monte Carlo simulated lung SPECT. Phys Med Biol. 1998;43:2325-2336.

15. Gustafsson A, Jacobsson L, Johansson A, Moonen M, Tylen U, Bake B. Evaluation of various attenuation corrections in lung SPECT in healthy subjects. Nucl Med Commun. 2003;24:1087-1095.

16. Petersson J, Sánchez-Crespo A, Larsson SA, Mure M. Physiological imaging of the lung: single-photon-emission computed tomography (SPECT). J Appl Physiol. 2007;102:468-476.

17. Links JM, Becker LC, Rigo P, et al. Combined corrections for attenuation, depthdependent blur, and motion in cardiac SPECT: a multicenter trial. J Nucl Cardiol. 2000;7:414-425.

18. Mariani G, Bruselli L, Kuwert T, et al. A review on the clinical uses of SPECT/ CT. Eur J Nucl Med Mol Imaging. 2010;37:1959-1985. 